\title{
Renditen und Volatilität bei Ein-/Ausstiegsstrategien
}

\author{
Prof. Dr. Gregor Dorfleitner, Wien, und Dipl.-Kfm. Christian Klein, Augsburg
}

\begin{abstract}
In der Praxis wird oft versucht, mit Hilfe von Ein-/Ausstiegsstrategien bei der Investition in eine Aktie Überrenditen zu erzielen und/oder die Volatilität des Engagements zu verringern. Wir bezeichnen hier das gezielte, von einem externen Signal indizierte, (Wieder-)Einoder Aussteigen in ein einzelnes, ausgesuchtes Investment als „Market Timing“ und zeigen aus finanzmathematischer Sicht, wie die periodenbezogenen Renditen bei Market Timing berechnet werden können.
\end{abstract}

Prof. Dr. Gregor Dorfleitner ist Professor für Finanzierung an der Wirtschaftsuniversität Wien. Bevorzugte Forschungsgebiete: Risikomanagement, Statistik, Derivate.

Dipl.-Kfm. Christian Klein ist wissenschaftlicher Mitarbeiter am Lehrstuhl für Statistik der Universität Augsburg. Bevorzugte Forschungsgebiete: Behavioral Finance, Statistik, Kapitalmarkteffizienz.

\section{Motivation}

Nach der Entscheidung für eine Anlagemöglichkeit, z. B. eine bestimmte Aktie oder ein bestimmter Aktienindex, bleibt es dem Investor überlassen, ob er für die Dauer seiner Investition in seine Anlage investiert bleibt (der Buyand-Hold-Fall) oder ob er durch Desinvestition und Wiedereinstieg in die Anlage versuchen will, eine höhere Rendite als die Buy-and-Hold-Rendite zu erzielen. Voraussetzung für die systematische Generierung von Überrenditen ist ein geeignetes Prognoseinstrument für die Wertentwicklung der Anlage. Die Existenz von solchen Prognoseinstrumenten wäre allerdings nicht mit der Effizienzmarkttheorie vereinbar, vgl. z. B. Fama (1970). Während die wissenschaftliche Welt diskutiert, ob solche Prognoseinstrumente überhaupt existieren können, ist in der Praxis dieser systematische Ein-/Ausstieg in eine Investition (im Folgenden als Market Timing bezeichnet) durchaus üblich. Um Signale für den Ein- und Ausstieg zu erhalten, wird beispielsweise die fundamentale oder technische Analyse verwendet. Eine Reihe von empirischen Untersuchungen beschäftigt sich mit der Performance der technischen Analyse die Rendite betreffend, siehe z. B. Herlitz (1975), Hofmann (1973), Lo/Mamaysky/Wang (2000) oder Dorfleitner/Klein (2002), mit sowohl negativen als auch positiven Ergebnissen.

Um ex post die Performance einer Ein-/Ausstiegsstrategie (im Folgenden Market-Timer-Strategie) bewerten $\mathrm{zu}$ können, ist sowohl die Berechnung der Rendite als auch der Volatilität notwendig. In den folgenden Abschnitten demonstrieren wir die teilweise nicht ganz triviale Herleitung der Gesamt- und Periodenrenditen einer Market-Timer-Strategie.
Der Einfluss von Market Timing auf die Volatilität einer Investition wird in der Praxis als günstig im Sinne einer Reduktion der Volatilität angenommen. Grund hierfür ist der ,glättende“ Effekt des Market Timings. Abb. 1 zeigt diesen Effekt anhand eines (fiktiven) Renditeverlaufs. Die kumulierte Rendite der Market-Timer-Strategie verläuft „flacher“ als die zugehörige Buy-and-Hold-Rendite, da der Investor zeitweise desinvestiert ist und seine kumulierte Rendite in diesem Zeitraum konstant bleibt. Wir zeigen, wie die Volatilität bei Market Timing berechnet wird. Dies ist aus risikotheoretischer Sicht nötig, um den Einfluss einer Market-Timer-Strategie auf die Investition bestimmen zu können. Die Volatilität wird beispielsweise benötigt, um das Risiko einer Investition abschätzen zu können. Darüber hinaus diskutieren wir den oben beschriebenen Effekt der Volatilitätsreduktion und demonstrieren, dass aus finanzmathematischer Sicht der erwünschte Effekt der Volatilitätsreduktion nicht, wie häufig angenommen, in jedem Fall eintreten muss, sondern dass sogar die Gefahr einer Erhöhung der Volatilität durch das Market Timing besteht.

Wir gehen dabei wie folgt vor: In Abschnitt 2 definieren wir die möglichen Vorgehensweisen eines Market Timers und betrachten die zugehörige Benchmark, die Buy-andHold-Strategie, sowie deren Renditen und Volatilität. Abschnitt 3 untersucht die verschiedenen Investitionsmöglichkeiten eines Market Timers bezüglich deren Einfluss auf die Volatilität. Abschnitt 4 enthält ein kurzes Beispiel zu der hier besprochenen Thematik.

\section{Market Timing}

Ein Market Timer ist ein Investor, welcher versucht über aktives Handeln mit seinem Investment Auf- und Abwärtsphasen des Marktes auszunutzen. Sein Ziel dabei ist es, entweder Überrenditen zu erzielen und/oder sein Risiko zu verringern. Im Gegensatz zu einem Buy-and-HoldInvestor verkauft er, nachdem er sich für eine Anlagemöglichkeit entschieden hat, seine Investition zeitweise, um schwache Marktphasen zu vermeiden. Die Diskussion über den Erfolg und den Sinn von Market Timing findet vor allem im Zusammenhang mit der Bewertung von gemanagten Fonds statt. Erfolgreiche Fonds-Manager genießen den Ruf, über besondere Market-Timing-Fähigkeiten zu verfügen. Die Wissenschaft hat sich ausführlich mit dieser Thematik beschäftigt (vgl. Treynor/Mazuy, 1966; Merton 1981; Henriksson/Merton, 1981). Im Gegensatz zu dieser Literatur beschäftigen wir uns allerdings mit den Auswirkungen von Market Timing auf die Volatilität eines einzelnen Investments.

Da wir in dieser Arbeit auf das reine Market Timing fokussieren, wie es etwa auch von Tradern praktiziert wird, ge- 


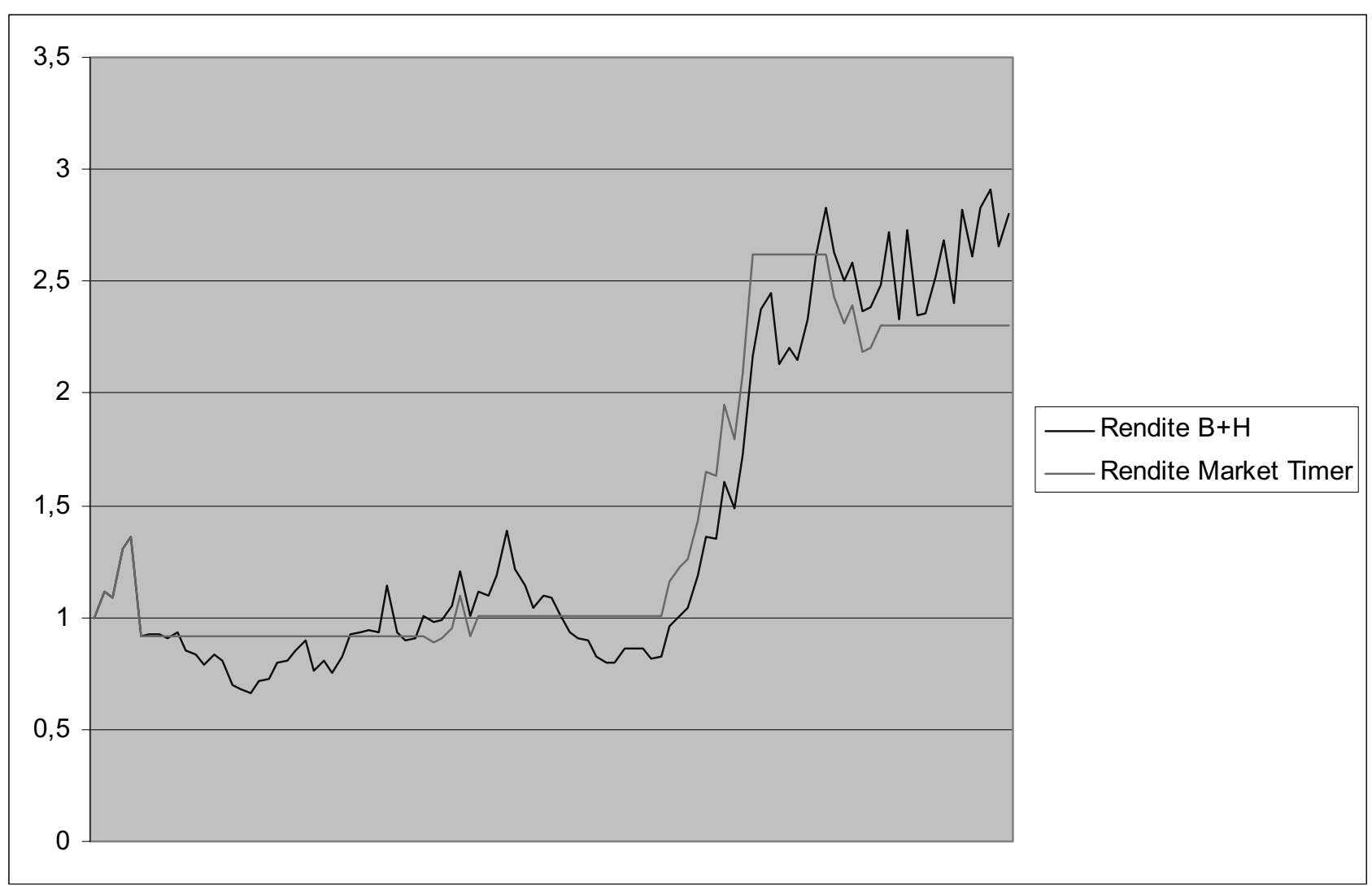

Abb. 1: Beispiel für die glättende Wirkung einer Market-Timer-Strategie

hen wir davon aus, dass zu Beginn jeder Periode Handelssignale vorliegen, und zwar solche, die entweder zum Kaufen, Halten oder Verkaufen raten. Eine feinere Unterteilung (,starkes Kaufsignal“, „,schwaches Kaufsignal“ etc.) wäre denkbar, soll an dieser Stelle aber nicht durchgeführt werden. Das Signal kann auf mehrere Weisen generiert werden. Eventuell richtet sich der Investor nach den Ratschlägen seines Bankberaters oder eines Aktienjournals. Alternativ könnte der Investor die fundamentale, die technische oder eine andere Art der Aktien- oder Marktanalyse anwenden, um ein Kauf- oder Verkaufssignal zu erhalten. Im Folgenden setzen wir die Existenz der Signale voraus. Nachdem er das Signal erhalten hat, sind - abhängig von seiner momentanen Situation (investiert oder nicht investiert) - mehrere Handlungsalternativen möglich. Tab. 1 enthält ein beispielhaftes Set an Handlungsalternativen.

Der Investor wird also nach Tab. 1, bei einem Halten-Signal sein Anlageobjekt behalten, sofern er investiert ist. Alternativ wäre in dieser Situation denkbar, dass er es verkauft, da er besonders risikoscheu ist und Halten bereits als ein für ihn ungünstiges Signal interpretiert.

\begin{tabular}{|c|c|c|}
\hline \multirow{2}{*}{ Signal } & \multicolumn{2}{|c|}{ Zustand } \\
\cline { 2 - 3 } Kauf & nicht investiert & investiert \\
Verkauf & kaufen & nichts tun \\
Halten & nichts tun & verkaufen \\
& nichts tun & nichts tun \\
\hline
\end{tabular}

Tab. 1: Investitionsentscheidungen in Abhängigkeit von Zustand und Signal
Bei einem Einstieg in den Markt, also dann, wenn der Market Timer die Alternative Kaufen wählt, existieren mehrere mögliche Strategien, dies zu tun. Die Auswahl der Strategie hat dabei einen bedeutsamen Einfluss auf die Volatilität des Investments. Wir unterscheiden zwischen vier Möglichkeiten, neben denen noch weitere denkbar sind:

- Reinvesting: Der Anleger investiert bei jedem Engagement seine gesamte bis dahin erzielte Anlagesumme.

- Constant Proportion: Der Anleger investiert bei jedem Engagement einen konstanten Anteil seiner bis dahin aufgelaufenen Anlagesumme, der Rest bleibt auf dem Konto.

- Rebalancing: Der Anleger investiert bei jedem Engagement einen fixen Betrag. Die Differenz zu der bis dahin aufgelaufenen Zwischensumme legt er auf ein Konto bzw. nimmt er als Kredit auf.

Als vierte Möglichkeit führen wir die Short-Strategie ein, welche die zusätzliche Option beinhaltet, short zu gehen, also auf eine negative Renditeentwicklung des Investments zu spekulieren. Dabei baut die Strategie auf eine der oben genannten Strategien auf. Wir verwenden hier eine Erweiterung der Reinvesting-Strategie.

Wir betrachten einen Zeitraum, der in $n$ hintereinander liegende Perioden zerfällt. Dies könnte beispielsweise ein Jahr sein, das aus $n=52$ Wochen besteht. Zu jedem der Zeitpunkte $t=0, \ldots, n$ gibt es einen Kurs $K_{t}$ des Investmentobjekts. Die zur Periode $t$ gehörige Rendite definieren wir als: 
$R_{t}=\frac{K_{t}-K_{t-1}}{K_{t-1}}=\frac{K_{t}}{K_{t-1}}-1$ mit $t=1, \ldots, n$.

Hier wird der Kurszuwachs von der Vorperiode $t$ - 1 bis zur Periode $t$ ins Verhältnis gesetzt zum Kurs der Vorperiode. Wir entscheiden uns damit für den so genannten diskreten Renditebegriff, da dieser die Eigenschaft der Portfolioadditivität besitzt, welche weiter unten noch von Bedeutung sein wird. Die stetige Rendite böte im Gegensatz dazu eine einfachere Handhabung bei der Aggregation über die Zeit hinweg. Zu stetigen und diskreten Renditen siehe Dorfleitner (2002). Die hier ermittelten Renditen können leicht in stetige Renditen umgerechnet werden, indem man den Quotienten $\frac{K_{t}}{K_{t-1}}$ logarithmiert.

Die Rendite lässt sich auch bezogen auf einen längeren Zeitraum definieren. Seien $s$ und $t$ zwei Zeitpunkte mit $s<t$. Dann ist die Rendite von $s$ bis $t$ definiert als:

$R_{s, t}=\frac{K_{t}-K_{s}}{K_{s}}=\frac{K_{t}}{K_{s}}-1$.

Offensichtlich gilt der Zusammenhang:

$R_{s, t}=\prod_{i=s+1}^{t}\left(R_{i}+1\right)-1$.

Den Quotienten $\frac{K_{t}}{K_{s}}$ bezeichnen wir auch als Wachstumsfaktor. Für diesen gilt mit (2) und (3):

$\frac{K_{t}}{K_{s}}=\prod_{i=s+1}^{t}\left(R_{i}+1\right)$.

Anders herum kann man eine einperiodige Rendite auch mithilfe der Wachstumsfaktoren ab einem bestimmten Zeitpunkt $s$ ausdrücken:

$R_{t}=\frac{K_{t}}{K_{t-1}}-1=\frac{\frac{K_{t}}{K_{s}}}{\frac{K_{t-1}}{K_{s}}}-1=\frac{R_{s, t}+1}{R_{s, t-1}+1}-1$.

Dieser Zusammenhang macht die Berechnung zunächst komplizierter, er wird jedoch weiter unter noch benötigt.

Beispiel: Zur Illustration werden die gerade vorgestellten Erkenntnisse in einem Beispiel veranschaulicht. Wir betrachten eine Investition in eine Aktie. Tab. 2 zeigt den Kursverlauf der Aktie. In der zweiten Zeile ist das Signal des Market Timers dargestellt, welches er in Periode $t$ erhält. Er reagiert auf dieses Signal mit den Strategien aus Tab.1. Seine möglichen Zustände sind „Nicht Investiert“ (-) oder „Investiert“. Betrachten wir als Beispiel den Zeitpunkt $t=0$. Der Anleger ist nicht investiert und erhält das Signal „Kauf“. Er erwirbt die Aktie für 100. Bei einem Kurs von 84 Punkten ist der Anleger im Besitz der Aktie, er ist investiert. Mit Formel (1) berechnen wir die Periodenrenditen:

\begin{tabular}{|c|c|c|c|}
\hline $\mathrm{t}$ & 0 & 1 & 2 \\
\hline Kurs & 100 & 84 & 91 \\
Signal & Kauf & Halten & Verkauf \\
Zustand & - & Investiert & Investiert \\
\hline
\end{tabular}

Tab. 2: Beispiel
$R_{1}=\frac{84}{100}-1=-0,160$ und $R_{2}=\frac{91}{84}-1=0,083$.

Die kumulierte Rendite von Periode 0 bis 2 beträgt nach Formel (2):

$R_{0,2}=\frac{91}{100}-1=-0,090$

oder nach Formel (3):

$R_{0,2}=0,84 \cdot 1,083-1=-0,090$.

Um nun die oben erwähnten Strategien besser beurteilen zu können, benötigen wir einen Vergleichsmaßstab, eine Benchmark. Als solche wird üblicherweise die Renditeentwicklung einer Buy-and-Hold-Strategie über den identischen Zeitraum hergenommen. Der Investor bleibt während der gesamten Zeit $t=0, \ldots, n$ investiert. Die Gesamtrendite des Buy-and-Hold-Investors beträgt

$R_{0, n}=\frac{K_{n}}{K_{0}}-1=\prod_{i=1}^{n}\left(R_{i}+1\right)-1$.

Dabei ist $\mathrm{K}_{0}$ der Wert oder Kurs der Investition zum Zeitpunkt 0 und $K_{n}$ der entsprechende Wert am Ende des Investitionszeitraumes. Unser Fokus liegt auf der Entwicklung der Volatilität oder der Standardabweichung des Investments. Um diese bestimmen zu können, benötigen wir die einzelnen Periodenrenditen $R_{t}$, also die Renditen der betrachteten äquidistanten Zeitabschnitte. Die Länge der einzelnen Zeitperioden spielt dabei für unsere Betrachtungen keine Rolle. Sie sind abhängig von dem Planungshorizont des Anlegers. Agiert der Market Timer beispielsweise maximal einmal pro Tag, sind für die Berechnung der Volatilität seiner Anlage die jeweiligen Tagesrenditen notwendig. Für einen Daytrader hingegen können Minuten oder Viertelstunden die relevanten Zeitintervalle definieren. Im Falle der Buy-and-Hold-Strategie sind die Periodenrenditen schlicht gleich $R_{t}$.

Mit den Periodenrenditen berechnen wir die Standardabweichung $\hat{\sigma}$ des Investments, wobei es sich hier korrekter formuliert um eine Schätzung handelt, wenn die Periodenrenditen als realisierte Zufallszahlen angesehen werden:

$\hat{\sigma}=\sqrt{\frac{n \sum_{t=1}^{n} R_{t}^{2}-\left(\sum_{t=1}^{n} R_{t}\right)^{2}}{n(n-1)}}$.

Die nach Formel (7) berechnete Volatilität bezieht sich auf den Zeitraum einer Periode. Die Berechnung der Periodenrenditen bei der Anwendung von Market-Timing-Strategien wird in den folgenden Abschnitten hergeleitet.

\section{Die Investitionsstrategien eines Market Timers}

Die von den Buy-and-Hold-Periodenrenditen im Allgemeinen abweichenden Periodenrenditen der Market-Timing-Strategien seien mit $\tilde{R}_{t}$ bezeichnet. Sind diese Renditen für alle Zeitpunkte $t=1, \ldots, n$ bekannt, dann kann die Gesamtrendite $\tilde{R}_{0, n}$ und die Volatilität $\hat{\tilde{\sigma}}$ berechnet werden. In den folgenden vier Abschnitten werden wir uns $\mathrm{zu}-$ 
nächst um die Herleitung der Renditen $\tilde{R}_{1}, \ldots, \tilde{R}_{n}$ bei bekannten Renditen $R_{1}, \ldots, R_{n}$ des Investitionsobjektes bemühen.

\subsection{Reinvesting}

Unter Reinvesting verstehen wir, dass ein Investor bei jedem Wiedereintritt in den Markt sein gesamtes, bis dahin kumuliertes Anlagekapital investiert. Der Market Timer verkauft also zu einem bestimmten Zeitpunkt sein Investment, parkt den Erlös auf einem Konto und setzt später bei der Wiederanlage das gesamte Kapital, welches auf dem Konto liegt, für den Wiedererwerb seiner Anlage ein. Vereinfachend gehen wir hier davon aus, dass dies ohne Probleme möglich ist, also dass beispielsweise Aktien beliebig teilbar sind und somit eine fixe Summe für den (Wieder-)Kauf komplett investiert werden kann.

Der Market Timer ist in diesem Fall mit zwei alternativen Situationen konfrontiert. Ist er investiert, so sind seine Renditen der einzelnen Perioden identisch mit den Renditen des Buy-and-Hold-Anlegers. Hat er den Markt verlassen, erfährt er eine Rendite von null, vorausgesetzt (und davon gehen wir aus) sein Konto, auf dem sein Geld geparkt ist, wird nicht verzinst. Formal ausgedrückt heißt das:

$\tilde{R}_{t}=\left\{\begin{array}{l}R_{t}, \text { wenn investiert } \\ 0, \text { sonst. }\end{array}\right.$

Die Berechnung der Volatilität erfolgt nun analog zu Formel (7).

\subsection{Short-Strategie}

Ein Market Timer stellt bei der bisher betrachteten Strategie die Investition dann glatt, wenn er einen negativen Kursverlauf in den nächsten Perioden vermutet. Er unternimmt jedoch nichts, falls er nicht bereits investiert ist. Eine denkbare Alternative zu dieser Vorgehensweise wäre, auf eine negative Kursentwicklung zu spekulieren, also auf das Investment short zu gehen, d.h. es zu verkaufen, auch wenn man es gar nicht besitzt. Dies ist eine Erweiterung der soeben dargestellten Reinvestment-Strategie. Zusätzlich zu den Optionen ,,investiert“ und ,nicht investiert" führen wir die Option ,short investiert“ ein. Auf ein Investmentobjekt short zu gehen ist in der Realität unterschiedlich schwer: Handelt es sich etwa um einen Aktienindex, auf den ein Future gehandelt wird (z. B. den $D A X$ oder den DJ EURO STOXX), dann ist es denkbar einfach auf den Index short zu gehen, indem man eine Short-Position in dem Future eröffnet oder ein entsprechendes ShortZertifikat kauft. (Wegen einer Einführung in das Gebiet der Futures siehe Hull, 2000; zu Short-Zertifikaten Baule/ Scholz/Wilkens, 2004.) Bei einzelnen Aktien hingegen kann es sehr schwierig sein, Leerverkäufe zu tätigen. Auf derartige technischen Details wollen wir hier nicht eingehen.

Ein Problem das sich dabei in jedem Falle stellt, ist, dass im Zeitpunkt des Shortgehens eigentlich kein Geld investiert wird, sondern im Gegenteil vielmehr ein gewisser Betrag vereinnahmt wird. Um in der Realität Shortgehen zu

\section{Das Standardwerk in Neuauflage!}

\author{
Perridon/Steiner
}

\section{Finanzwirtschaft der Unternehmung}

Von Prof. Dr. Louis Perridon, München, und

Prof. Dr. Manfred Steiner, Augsburg

13., überarbeitete und erweiterte Auflage. 2004

XXXIII, 732 Seiten. Gebunden $€$ 25,-

ISBN 3-8006-3112-1 (Erscheint Anfang Oktober 2004)

Theoretische Ansätze der Finanzierung haben für die finanzwirtschaftliche Praxis zunehmend an Bedeutung gewonnen. Dieser Entwicklung trägt dieses Buch Rechnung. Die Verbindung von Theorie und institutionellen Fragestellungen sowie Rahmenbedingungen kennzeichnet auch diese Auflage.

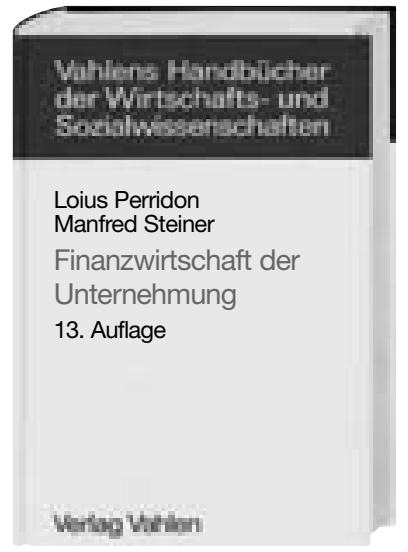
Sie liefert einen fundierten Überblick über das Management der Vermögensstruktur, Wertpapiergeschäfte, Alternativen der Kapitalaufbringung, Finanzanalyse und Finanzplanung.

\section{Wesentliche Neuerungen in Stichwörtern:}

Discounted-Cash-Flow-Verfahren zur fundamentalen Unternehmensbewertung • Kreditderivate - Ablauf des Insolvenzverfahrens • Bilanzierung von Leasing nach IFRS und US-GAAP • moderne Prognoseverfahren.

\section{FAX-COUPON}

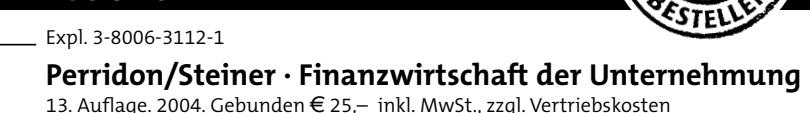

13. Auflage. 2004. Gebunden $€ 25,-$ inkl. MwSt., zzgl. Vertriebskosten

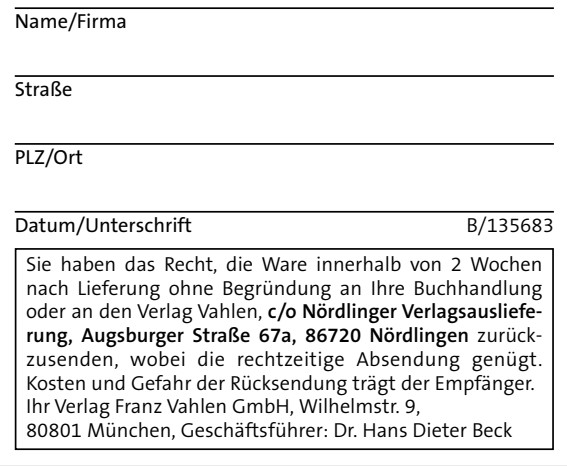
oder beim

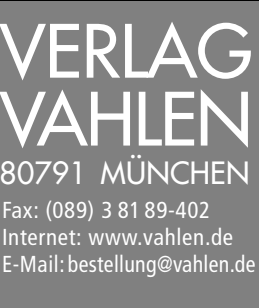


können, muss allerdings eine Sicherheitsleistung hinterlegt, d.h. investiert, werden. Wir nehmen für alles Folgende an, dass diese Leistung genau so groß ist, wie der Wert des Investments zu dem Zeitpunkt. Investiert man auf diese Weise zum Zeitpunkt $s$ beim Kurs $K_{s}$ und stellt man die Position zum Zeitpunkt $t$ beim Kurs $K_{t}$ wieder glatt, so erreicht man die Rendite:

$\frac{K_{s}-K_{t}}{K_{s}}=1-\frac{K_{t}}{K_{s}}=-R_{s, t}$

Mit einer derart spezifizierten Short-Investition kann ein Investor also exakt den negativen Renditewert einer normalen Long-Investition erzielen. Dies gilt für die gesamte Rendite der Short-Investition vom Zeitpunkt $s$ bis $t$. Für die Berechnung der Volatilität sind jedoch die Periodenrenditen von Belang. Dazu muss man im Grunde den Kontostand zum Ende jeder Periode nachbilden und daraus Renditen berechnen. Ist man long oder nicht investiert, so ergeben sich dieselben Renditen wie bei der ReinvestingStrategie im vorigen Abschnitt. Im Falle des Shortgehens zum Zeitpunkt $s$ haben wir in Formel (9) oben gezeigt, wie die Renditen bis zum Zeitpunkt $t$ berechnet werden können. Die Perioden-Rendite vom Zeitpunkt $t-1$ bis $t$ ergibt sich als Kontostand zum Zeitpunkt $t$ durch Kontostand zum Zeitpunkt $t-1$ abzüglich 1 . Nun ist der Kontostand aber direkt proportional zum Wachstumsfaktor vom Zeitpunkt $s$ aus gesehen. Der Wachstumsfaktor ergibt sich als Renditewert plus 1 . Wir erhalten also die Periodenrendite beim Shortgehen als

$\frac{1-R_{s, t}}{1-R_{s, t-1}}-1=\frac{2-\frac{K_{t}}{K_{s}}}{2-\frac{K_{t-1}}{K_{s}}}-1=\frac{2-\prod_{i=s+1}^{t}\left(R_{i}+1\right)}{2-\prod_{i=s+1}^{t-1}\left(R_{i}+1\right)}-1$.

Für die erste Gleichheit benötigen wir Formel (2), für die zweite Formel (4). Der letztere Term erscheint zwar komplizierter als die beiden anderen. Er hat jedoch einen entscheidenden Vorteil: Es kommen darin nur die Basis-Periodenrenditen des Investmentobjektes vor.

Zusammenfassend haben wir nun die Periodenrenditen der Short-Strategie wie folgt zu berechnen:

$\tilde{R}_{t}= \begin{cases}R_{t}, & \text { wenn investiert } \\ \frac{2-\prod_{i=s+1}^{t}\left(R_{i}+1\right)}{2-\prod_{i=s+1}^{t-1}\left(R_{i}+1\right)}-1, & \text { wenn short investiert seit } s<t(11) \\ 0, & \text { wenn nicht investiert. }\end{cases}$

Beispiel: Wieder verwenden wir zur Illustration das Beispiel einer Investition in eine Aktie. Tab. 3 zeigt hier den Kursverlauf der Aktie, analog zu vorherigem Beispiel. Zusätzlich gibt es hier für den Anleger die Option short zu gehen.

\begin{tabular}{|c|c|c|c|}
\hline $\mathrm{t}$ & 0 & 1 & 2 \\
\hline Kurs & 99 & 105 & 112 \\
Signal & Verkauf & Verkauf & Halten \\
Zustand & - & Short & Short \\
\hline
\end{tabular}

Tab. 3: Beispiel Short-Strategie
Mit Formel (11) berechnen wir die Periodenrenditen

$\tilde{R}_{1}=\frac{2-1,061}{1}-1=-0,061$

und

$\tilde{R}_{2}=\frac{2-1,061 \cdot 1,067}{2-1,061}-1=\frac{0,8679}{0,9390}-1=-0,0757$.

Die kumulierte Rendite von Periode 0 bis 2 beträgt nach Formel (9):

$\tilde{R}_{0,2}=1-\frac{112}{99}=-0,1313$

oder

$\tilde{R}_{0,2}=(-0,061+1) \cdot(-0,0757+1)-1=-0,1313$.

\subsection{Constant Proportion}

Ein beliebtes Verfahren bei der Portfolio-Bildung ist die so genannte Constant-Proportion-Methode. Das anzulegende Budget wird in festen Anteilen auf die Wertpapiere und die sichere Anlage aufgeteilt. In unserem Ein-Wertpapier-Fall investiert der Anleger bei jedem Einstieg einen konstanten Anteil $\lambda \in[0 ; 1]$ seines Kontostandes in das Investment. Während des Zeitraumes, in dem er investiert ist, passt er die Anteile (der Einfachheit halber) nicht an. Die Periodenrendite ist hier wieder mit der oben dargestellten Idee herzuleiten, dass der Kontostand bei einem Long-Investment seit Zeitpunkt $s$ direkt proportional zum Wachstumsfaktor seit $s$ ist. Im Zeitpunkt $s$ wird ein Anteil $\lambda$ in das Investmentobjekt investiert und der Restanteil von $1-\lambda$ in Cash gehalten. Da bei Verwendung der diskreten Rendite innerhalb von Portfolios die Additivitätseigenschaft für Renditen (und auch Wachstumsfaktoren) gilt, erhalten wir den Wachstumsfaktor bezogen auf das Intervall von $s$ bis $t$ :

$\lambda \frac{K_{t}}{K_{s}}+(1-\lambda) \cdot 1=\lambda \prod_{i=s+1}^{t}\left(R_{i}+1\right)+1-\lambda$

Da auch hier die Rendite im Falle des Nichtinvestierens gleich null ist, erhalten wir mit Formel (12) (und analog zu (5)) unter Ausnutzung der auch im vorigen Abschnitt erwähnten Proportionalität des Kontostandes zum Wachstumsfaktor die folgenden Periodenrenditen der ConstantProportion-Strategie:

$\tilde{R}_{t}= \begin{cases}\frac{\lambda \prod_{i=s+1}^{t}\left(R_{i}+1\right)+1-\lambda}{\lambda \prod_{i=s+1}^{t-1}\left(R_{i}+1\right)+1-\lambda}-1, & \text { wenn seit Zeitpunkt } \\ 0, & \mathrm{~s}<\mathrm{t} \text { investiert }\end{cases}$

Beispiel: Wir zeigen die Anwendung der Formel anhand des ersten Beispiels (Tab. 2). Der Anleger wählt eine Constant-Proportion-Strategie mit $\lambda=0,9$. Seine Rendite $\tilde{R}_{1}$ beträgt somit nach Formel (13):

$\tilde{R}_{1}=\frac{0,9 \cdot(-0,16+1)+1-0,9}{0,9 \cdot 1+1-0,9}-1=\frac{0,856}{1}-1=-0,144$

und 


$$
\begin{aligned}
\tilde{R}_{2} & =\frac{0,9 \cdot(-0,16+1) \cdot(0,083+1)+1-0,9}{0,9 \cdot(-0,16+1)+1-0,9}-1 \\
& =\frac{0,062748}{0,856}=0,0733 .
\end{aligned}
$$

Als Gesamtrendite erhalten wir mit Formel (12):

$\tilde{R}_{02}=0,9 \frac{91}{100}+0,1-1=-0,081$

oder nach Formel (3):

$(1-0,144) \cdot(1+0,0733)-1=-0,081$.

\subsection{Rebalancing}

Ein Investor, der nach der Rebalancing-Strategie investiert, setzt bei jedem Eintritt in den Markt einen konstanten Geldbetrag ein. Eine eventuelle Differenz zwischen der Summe, welche investiert wird, und der Summe, welche zur Verfügung steht (also den kumulierten Renditen) legt der Investor auf einem Cash-Konto an.

Die Berechnung der Periodenrenditen, die für die Bestimmung der Volatilität notwendig ist, ist nicht so trivial wie in Abschnitt 3.1., da hier die Entwicklung des Cash-Kontos berücksichtigt werden muss. Am ehesten kann man sich mit einer rekursiven Definition behelfen.

Für $t=1$ gilt:

$\tilde{R}_{t}= \begin{cases}R_{t}, & \text { wenn investiert } \\ 0, & \text { wenn nicht investiert. }\end{cases}$

Für alle weiteren Perioden muss man analog zu Constant Proportion den Wachstumsfaktor eines Portfolios berechnen, wobei die Gewichte nicht konstant sind, sondern in jedem Investitionszeitpunkt $s$ neu zu berechnen sind und von der bis dahin erwirtschafteten Gesamtrendite (bzw. vom Gesamtwachstumsfaktor) abhängen. Statt wie in Formel (12) mit $\lambda$ und $1-\lambda$ zu gewichten, sind die Gewichte nun

$\frac{1}{\prod_{i=1}^{s}\left(\tilde{R}_{i}+1\right)}$

für den in Aktien investierten Anteil und

$\frac{\prod_{i=1}^{s}\left(\tilde{R}_{i}+1\right)-1}{\prod_{i=1}^{t-1}\left(\tilde{R}_{i}+1\right)}$

für den in Cash investierten Anteil mit Wachstumsfaktor 1. Wie man sieht, addieren sich diese relativen Gewichte zu 1 auf. Diese Normierung ist zur Anwendung der Portfolioadditivität auch zwingend nötig.

Analog zu (12) erhalten wir nun:
$\frac{1}{\prod_{i=1}^{s}\left(\tilde{R}_{i}+1\right)} \cdot \prod_{i=s+1}^{t}\left(R_{i}+1\right)+\frac{\prod_{i=1}^{s}\left(\tilde{R}_{i}+1\right)-1}{\prod_{i=1}^{s}\left(\tilde{R}_{i}+1\right)} \cdot 1$.

Für $t>1$ gilt damit zusammenfassend:

$\tilde{R}_{t}= \begin{cases}\frac{\prod_{i=s+1}^{t}\left(R_{i}+1\right)+\prod_{i=l}^{s}\left(\tilde{R}_{i}+1\right)-1}{\prod_{i=s+1}^{t-1}\left(\mathrm{R}_{\mathrm{i}}+1\right)+\prod_{\mathrm{i}=t}^{-l}\left(\tilde{\mathrm{R}}_{\mathrm{i}}+1\right)-1}-1, & \text { wenn seit Zeitpunkt } \\ 0, & \text { wenn nicht investiert. }\end{cases}$

Dabei wurde der Term

$\frac{1}{\prod_{i=1}^{s}\left(\tilde{R}_{i}+1\right)}$

aus (15) in Zähler und Nenner der ersten Zeile gekürzt. Wenn der Investor nur eine einzelne Periode investiert war, vereinfacht sich die Formel auf

$\tilde{R}_{t}=\frac{R_{t}}{\prod_{i=1}^{t-1}\left(\tilde{R}_{i}+1\right)}$.

Die Gesamtrendite am Ende des Investitionszeitraumes setzt sich zusammen aus der Summe der diskreten Renditen der einzelnen Transaktionen:

$R=\sum_{i=1}^{m}\left(\frac{K_{s(i)}}{K_{b(i)}}-1\right)$.

$K_{s(i)}$ entspricht dem Kurs der Investition bei Ausstieg aus Transaktion $i, K_{b(i)}$ dem Kurs, der für den jeweiligen Wiedereinstieg in die Investition relevant war. Insgesamt wurden $m$ Transaktionen durchgeführt.

Man kann nun durch vollständige Induktion über $m$ zeigen, dass (18) gleich der aus den $\tilde{R}_{t}$ berechneten Gesamtrendite

$\tilde{R}_{0, n}=\prod_{i=1}^{n}\left(\tilde{R}_{i}+1\right)-1$

ist. Den Beweis auszuführen, ersparen wir dem Leser an dieser Stelle.

Beispiel: Wiederum geben wir den Kursverlauf einer Aktie vor (Tab. 4).

„Zustand“ gibt die Situation des Investors zu Beginn der Periode wieder. Der Anleger ist zu Beginn der Perioden 0 und 5 nicht investiert und kauft die Aktie, nachdem er ein „Kauf“-Signal erhalten hat, zum Preis von 100 bzw. 112. Zwischen den Perioden 2 und 4 ist er nicht inves-

\begin{tabular}{|c|c|c|c|}
\hline \multirow{2}{*}{ Signal } & \multicolumn{3}{|c|}{ Zustand } \\
\cline { 2 - 4 } Kauf & short & nicht investiert & long \\
Verkauf & haltellen & long gehen & halten \\
Halten & halten & short gehen & klatt stellen \\
heine Aktion & halten \\
\hline
\end{tabular}

Tab. 5: Investitionsentscheidungen bei einer Short-Strategie in Abhängigkeit von Zustand und Signal

\begin{tabular}{|c|c|c|c|c|c|c|}
\hline $\mathrm{t}$ & 0 & 1 & 2 & $(\ldots)$ & 5 & 6 \\
\hline Kurs & 100 & 84 & 91 & $(\ldots)$ & 112 & 123 \\
Signal & Kauf & Halten & Verkauf & $(\ldots)$ & Kauf & Kauf \\
Zustand & - & investiert & investiert & $(-)$ & - & investiert \\
\hline
\end{tabular}

Tab. 4: Beispiel Rebalancing 


\begin{tabular}{|c|c|c|c|c|c|c|}
\hline $\mathrm{t}$ & 0 & 1 & 2 & 3 & 4 & 5 \\
\hline Kurs & 100 & 84 & 91 & 99 & 105 & 112 \\
Signal & Kauf & Halten & Verkauf & Verkauf & Halten & Kauf \\
Zustand & - & investiert & investiert & n.i. & n.i. & n.i. \\
Bei Short-Strategie & - & long & long & n.i. & short & short \\
\hline
\end{tabular}

\begin{tabular}{|c|c|c|c|c|c|c|}
\hline $\mathrm{t}$ & 6 & 7 & 8 & 9 & 10 & 11 \\
\hline Kurs & 123 & 167 & 171 & 172 & 199 & 222 \\
Signal & Kauf & Kauf & Kauf & Halten & Verkauf & - \\
Zustand & investiert & investiert & investiert & investiert & investiert & n.i. \\
Bei Short-Strategie & n.i. & long & long & long & long & n.i. \\
\hline
\end{tabular}

Tab. 6: Rechenbeispiel: Kursverlauf einer Aktie, Signale und daraus resultierende Zustände

\begin{tabular}{|c|ccccc|}
\hline Strategie & Buy \& Hold & Reinvesting & Rebalancing & $\begin{array}{c}\text { Const. Prop. } \\
(0,9)\end{array}$ & Short Strategy \\
\hline Rendite & 1,220 & 0,617 & 0,687 & 0,561 & 0,279 \\
Volatilität & 0,120 & 0,124 & 0,132 & 0,112 & 0,130 \\
\hline
\end{tabular}

Tab. 7: Ergebnisse des Rechenbeispiels

tiert. Seine Periodenrendite der Periode 1 beträgt nach Formel (14)

$\tilde{R}_{1}=-0,160$.

Für Periode 2 verwenden wir Formel (16):

$\tilde{R}_{2}=\frac{(0,083+1) \cdot(-0,160+1)+1-1}{(-0,160+1)+1-1}-1=0,083$.

Die Periodenrendite für Periode 6 beträgt (wieder nach Formel (16)):

$\tilde{R}_{6}=\frac{(0,098+1)+(-0,160+1) \cdot(0,083+1) \cdot 1 \cdot 1 \cdot 1-1}{1+(-0,160+1) \cdot(0,083+1) \cdot 1 \cdot 1 \cdot 1-1}-1=0,108$

Man beachte, dass sich die Werte $\tilde{R}_{1}, \tilde{R}_{2}$ gegenüber $R_{1}, R_{2}$ nicht unterscheiden, während dies bei $\tilde{R}_{6}$ im Vergleich zu $R_{6}=0,09821$ durchaus der Fall ist. Dies liegt in der Abhängigkeit von der Vorgeschichte begründet, die im Zeitpunkt $t=0$ keine Rolle spielt, im Zeitpunkt $t=5$ jedoch durchaus.

\section{Anwendungsbeispiel}

Folgendes Beispiel wurde in den vorangegangenen Abschnitten auszugsweise bereits als Demonstration verwendet. Wir betrachten eine Investition in eine Aktie. Tab. 6 zeigt den Kursverlauf der Aktie. In der zweiten Zeile ist das Signal des Market Timers dargestellt, welches er in Periode $t$ erhält. Er reagiert auf dieses Signal mit den Vorgehensweisen aus Tab. 1, falls er die Reinvesting-, die Constant Proportion- oder die Rebalancing-Strategie verwendet. Basiert seine Vorgehensweise auf der Short-Strategie, so hat er weitere Optionen zur Auswahl und hält sich an die Vorgehensweisen aus Tab. 5. Betrachten wir beispiels- weise den Zeitpunkt $t=1$. Bei einem Kurs von 84 ist der Anleger im Besitz der Aktie, er ist investiert. Nun erhält er das Signal „Verkauf“ und verkauft die Anlage zu einem Preis von 91. Er ist zu Beginn der 3. Periode nicht investiert („n.i.“). Da ein weiteres Verkaufs-Signal eintrifft, bleibt er es auch, falls er eine der Strategien Reinvesting, Constant Proportion oder Rebalancing verwendet. Bei der Short-Strategie spekuliert er nun auf eine negative Kursentwicklung und geht short auf die Aktie. Sein Zustand ist somit „Short“" zum Zeitpunkt $t=4$.

Wir setzen diese Werte nun in die hier aufgezeigten Formeln ein und erhalten als Ergebnis die Werte aus Tab. 7.

In diesem Beispiel führt die Buy-and-Hold-Strategie zur höchsten Rendite. Interessanterweise ist die Volatilität der Buy-and-Hold Strategie nach der Constant-ProportionStrategie die zweitniedrigste - der Market Timer hätte also auch nach einer eventuellen Risikobereinigung eine schlechtere Performance als ein Investor, welcher nach der Buy-and-Hold-Strategie investiert. Dieses Beispiel widerlegt somit auch, dass Market Timing immer zwangsläufig $\mathrm{zu}$ einer Volatilitätsreduktion führen muss. Hier ist das Gegenteil der Fall - die Volatilität wird erhöht.

\section{Schlussbetrachtung}

Die vorangehenden Überlegungen zeigten in erster Linie, wie bei verschiedenen Market-Timing-Strategien die Periodenrenditen zu berechnen sind. Mit Formel (7) war damit auch die Volatilität sofort berechenbar. Damit wird ex post die Schwankung der Periodenrendite entlang eines Vermögensentwicklungspfades gemessen, wie etwa auch in der Fonds-Performancemessung üblich. Auf eine Annualisierung der Volatilität, d.h. das Umrechnen in einen entspre- 
chenden Jahreswert, wurde verzichtet, da dies bei diskreten Renditen nicht ohne weiteres möglich ist. Siehe hierzu auch Dorfleitner (2002).

Zusätzlich zu der hier betrachteten Volatilität wird eine Anlegerin, die für einen bestimmten Zeitraum $[0, n]$ investieren will, auch an der Standardabweichung der Gesamtrendite $\boldsymbol{R}_{\mathbf{0}, \boldsymbol{n}}$ interessiert sein. Diese Größe, die als das Risiko des gesamten Investitionsprozesses interpretiert werden kann, lässt sich jedoch aus dem einen vorliegenden Pfad nicht ermitteln - d.h. schätzen - , hängt aber klar mit der hier berechneten Volatilität zusammen: Es besteht ein positiver Zusammenhang. Denn die Gesamtrendite wird durch Aggregation der einzelnen Periodenrenditen per Formel (6) erzeugt. Damit ist bei Strategien, die eher mit einer niedrigen Volatilität einhergehen, auch eine niedrige Standardabweichung der Gesamtrendite $R_{0, n}$ zu erwarten.

Wichtig für die tatsächliche Performance des Market Timers ist natürlich die Qualität des Signalgenerators, welcher hier als Black Box betrachtet wurde. Diese Arbeit konzentrierte sich auf das finanzmathematische Problem der Rendite- und Volatilitätsberechnung bei Market Timing. Wir konnten dabei zeigen, dass Market Timing nicht generell zu niedrigen Volatilitäten führen muss. Klar sollte aber sein, dass dies sehr wohl der Fall sein kann. Der Anspruch von Signalgeneratoren (etwa technische AnalyseWerkzeuge) besteht indes darin, Überrenditen zu erzeugen, also eine höhere Rendite bei gleicher Volatilität oder die gleiche Rendite bei niedrigerer Volatilität. Dies bringt uns an den eingangs erwähnten Punkt der Informationseffizienz von Kapitalmärkten zurück. Die Existenz eines einfachen und über die Zeit gleichbleibenden Wegs zur Erzeugung von Überrenditen darf aber bezweifelt werden.

\section{Literatur}

Baule, R., H. Scholz, M. Wilkens, Short-Zertifikate auf Indizes. Bewertung und Analyse eines innovativen Retail-Produktes für Baissephasen, in: Zeitschrift für Betriebswirtschaft, 74. Jg. (2004), S. 315-338.

Dorfleitner, G., Stetige versus diskrete Renditen. Überlegungen zur richtigen Verwendung beider Begriffe in Theorie und Praxis, in: Kredit und Kapital, 35. Jg. (2002), S. $216-241$.

Dorfleitner, G., C. Klein, Kursprognose mit Hilfe der Technischen Analyse. Eine empirische Untersuchung, in: Financial Markets and Portfolio Management, 16. Jg. (2002), 497 - 521.

Fama, E.F., Efficient Capital Markets. A Review of Theory and Empirical Work, in: Journal of Finance, 25. Jg. (1970), S. $383-$ 417.

Henriksson, R.D., R.C. Merton, On market Timing and investment Performance. II. Statistical Procedures for Evaluating Forecasting Skills, in: Journal of Business, 54. Jg. (1981), S. 513 - 533.

Herlitz, K., Kritische Analyse der Anwendung von Chart-Theorien zur Kursprognose deutscher Standardwerte, Dissertation der Freien Universität Berlin 1975.

Hull, J., Options, Futures, and Other Derivative Securities, 4. Aufl., Englewood Cliffs 2000.

Hofmann, H.W., Empirische Untersuchung verschiedener Anlagestrategien der Technischen Analyse anhand von 100 deutschen Standardaktien über einen Zeitraum von fünf Jahren (19671972) und Vergleich mit einer durch Simulation ermittelten Zufallsauswahl bzw. einer Kauf- und Halte-Strategie, Dissertation der Ludwigs-Maximilians-Universität München 1973.

Lo, A.W., H. Mamaysky, J. Wang, Foundations of Technical Analysis, Computational Algorithms, Statistical Inference, and Empirical Implementation, in: Journal of Finance, 55. Jg. (2000), S. $1705-1765$.

Merton, R.C., On market Timing and Investment Performance. I. An equilibrium theory of value for market forecasts, in: Journal of Business, 54. Jg. (1981), S. 363 - 360.

Treynor, J., F. Mazuy, Can mutual funds outguess the market?, in: Harvard Business Review, 44. Jg. (1966), S. 131 - 136.

\section{WiSt}

Schriftleitung: Verantwortliche Redakteure: für Betriebswirtschaftslehre Prof. Dr. Michael Lingenfelder, Universität Marburg, FB02, BWL III, Universitätsstraße 24, 35032 Marburg, Telefon: 06421/282 37 63; für Volkswirtschaftslehre Prof Dr. Norbert Berthold, Universität Würzburg, Sanderring 2 97070 Würzburg, Telefon: 0931/31 29 25. Mitarbeiter: Dipl. Kffr. Ines Bott, Marburg. E-Mail: bott@ wiwi.uni-marburg.de, Dipl.-Volkswirtin Irene Riegelsberger, Würzburg, E-mail: irene.riegelsberger@mail.uni-wuerzburg.de.

Mit der Annahme eines Manuskripts zur Veröffentlichung überträgt der Autor dem Verlag das ausschließliche Verlagsrecht für die Zeit bis zum Ablauf des Urheberrechts. Eingeschlossen sind insbesondere auch die Befugnisse zu Einspeicherung in eine Datenbank sowie das Recht de weiteren Vervielfältigung zu gewerblichen Zwecken im Wege enes photomechanischen oder eines anderen Verfahrens. Dem Autor verbleibt die Befugnis, nach Ablauf eines Jahres anderen Verlagen eine einfache Abdruckgenehmigung zu erteilen; ein Honorar hieraus steht dem Autor zu.

Urheber- und Verlagsrechte: Alle in dieser Zeitschrift veröffentlichten Beiträge sind urheberrechtlich geschützt. Das gilt auch für die veröffentlichten Gerichtsentscheidungen und ihre Leitsätze, denn diese sind geschützt, soweit sie vom Einsender oder von der Schriftleitung erarbeitet oder redigiert worden sind. Der Rechtsschutz gilt auch gegenüber Datenbanken und ähnlichen Einrichtungen. Kein Teil dieser Zeitschrift darf außerhalb der engen Grenzen des Urheberrechtsgesetzes ohne schriftliche Genehmigung des Verlags in irgendeiner Form - durch Fotokopie, Mikrofilm oder andere Verfahren - reproduziert oder in eine von Maschinen, insbesondere von Datenverarbeitungsanlagen verwendbare Sprache, übertragen werden.

Anzeigenabteilung: Verlag C.H. Beck, Anzeigenabteilung, Wilhelmstr. 9, 80801 München; Postanschrift: Postfach 4003 40, 80703 München

Media-Service: Telefon: 089/381 89-781, Telefax: 089/ 381 89-782, media-service@ beck.de

Media-Disposition (Herstellung Anzeigen, technische Daten): Telefon: 089/381 89-598/-603, Telefax Auftragsservice: 089/381 89-58

Verantwortlich für den Anzeigenteil: Fritz Lebherz Z.Zt. gilt Anzeigenpreisliste Nr. 25.

Verlag: C.H. Beck oHG, Wilhelmstraße 9, 80801 München, Telefon: 089/381 89-0, Telex: 5215085 beck d. Telefax: 089/38 1893 98, Postbank: München, Kto. 6229-802, BLZ 70010080 .

Erscheinungsweise: Monatlich.

Bezugspreise 2004: Halbj. € 55,- (darin € 3,60 MwSt.),
Vorzugspreis für Studenten (fachbezogener StudienVorzugspreis für Studenten (fachbezogener Studien
gang, gegen Nachweis) $€ 39,50$ (darin $€ 2,58$ MwSt.). gang, gegen Nachweis) $€ 39,50$ (darin $€ 2,58 \mathrm{MwSt}$.).
Einzelheft: $€ 9,80$ (darin $€ 0,64 \mathrm{MwSt}$.) jeweils zuzüglich Versandkosten.

Nicht eingegegangene Exemplare können nur innerhalb von 6 Wochen nach dem Erscheinungstermin reklamiert werden.

Bestellungen nehmen entgegen: jede Buchhandlung und der Verlag.

Abo-Service: Tel.: 089/381 89-679. Fax: 089/381 89-297. E-Mail: abo.service@beck.de.

Abbestellungen müssen 6 Wochen vor Halbjahresschluss erfolgen.

Adressenänderungen: Teilen Sie uns rechtzeitig Ihre Adressenänderungen mit. Dabei geben Sie bitte neben dem Titel der Zeitschrift die neue und die alte Adresse an. Hinweis gemäß $§ 7$ Abs. 5 der Postdienst-Datenschutzverord-

nung: Post AG dem Verlag die neue Anschrift auch dann mitteilen, wenn kein Nachsendeantrag gestellt ist. Hiergegen kann der Bezieher innerhalb von 14 Tagen nach Erscheinen dieses Heftes beim Verlag widersprechen.

Satz: FotoSatz Pfeifer GmbH, 82166 Gräfelfing.

Druck: Druckerei C.H. Beck, Bergerstraße 3, 86720 Nördlingen. 